\title{
The Human Microbiome Concept of Disease Prevention and Treatment: A Giant Leap in Medical Genetics
}

Sandeep Kumar Kar

Department of Cardiac Anaesthesiology, Institute of Postgraduate Medical Education and Research, Kolkata, India

"Corresponding author: Kar SK, Assistant Professor, Department of Cardiac Anaesthesiology, Institute of Postgraduate Medical Education and Research, 242, AJC Bose Road Kolkata: 700020, India, Tel: +91-9477234900; E-mail: sndpkar@yahoo.co.in

Rec Date: 04 Apr, 2016; Acc Date: 05 Apr, 2016; Pub Date: 09 Apr, 2016

Copyright: (c) 2016 Kar SK, This is an open-access article distributed under the terms of the Creative Commons Attribution License, which permits unrestricted use, distribution, and reproduction in any medium, provided the original author and source are credited.

Citation: Kar SK (2016) The Human Microbiome Concept of Disease Prevention and Treatment: A Giant Leap in Medical Genetics. Hereditary Genet 5: e114. doi: $10.4172 / 2161-1041.1000 \mathrm{e} 114$

\section{Editorial}

Human beings are holobionts; wherein diverse group of human cells and microbial cells function together in elaborate symbiosis. The total number of microbial cells exceeds the number of human cells 10 fold. Each healthy human being is estimated to harbour about 1 million microbial genes, while the human genome has only 20000 genes [1]. The microbes in human body can function as symbionts, commensals and sometimes even as pathogens. The ecological concept that is emerging now is that pathogens do not function in isolation, rather their emergence; invasion and effect on the host organ systems reflect interaction with other members of the microbial community. Sometimes multiple microbes in the human micro biome conspire to manifest pathogenicity and cause disease in human organ systems.

The speciality of Metagenomics is going to play a pivotal role in understanding human disease and plan appropriate interventions in the current era of Probiotics. Metagenomics represents a confluence of experimental and computational advances in genetic sciences and seem to envision medical microbiology from ecological viewpoint, according to which the functions of a given microbial organism and its impact on human organ systems depend on the context of other microbes harbouring the same community. Metagenomics skips the step of culturing and isolating individual microbes and leaps to the step of sequencing the DNA isolated directly from an investigational microbial community.

The microbiome concept of disease causation provides insight to new ways of classifying disease states and tries to find, whether the variability in structural and functional configuration of microbial communities are a cause or a consequence of the disease. There is perpetual dynamic interexchange of DNA within the human genome and the micro biome genome. Scientists or the researchers need to keep in his armaments the desirable genetic expressions expressed as effective translated protein (hormones or biomolecules) so as to glorify the human genome with desirable effects through suitably transgenetic mediated bacteriophages [2].

The microbiome conceptualises of a human genetic potential from a single set of genes, acquired at birth, increasing in number to a microbiome with additional genes, which are in dynamic equilibrium with microbial genes endowing us with favourable effects via a process influenced by our lifestyle and more specifically our diet.

There is evidence that westernization is accompanied with loss of bacterial species diversity in the microbiota and this loss with diet and lifestyle modification actually increase the vulnerability to chronic diseases of the developed world termed as Western diseases. This has led to the revolutionary era of designing probiotics which serve as a new approach to disease prevention and treatment opening the speciality of regenerative medicine. Probiotic medication therapy involves capsulated administration of microbial species to individuals harbouring communities of microbial communities that have not developed into a mature fully functional state or that have been disturbed during infections and environmental disasters and epidemics.

Probiotics assist in digestion and boost the body's immunity in diarrhoea and infections. There are probiotics that confer beneficial effect on the cardiovascular system, a specific strain of Lactobacillus called Lactobacillus reuteri $\mathbf{3 0 2 4 2}$ has been found to work in two distinct ways to lower cholesterol: It removes excess cholesterol from the body and increases the metabolism of cholesterol [3] Clinical studies demonstrate that Lactobacillus reuteri effectively lowers levels of total and LDL-cholesterol, while driving down inflammation and reducing other metabolic disturbances that raise cardiovascular risks. A safe, natural probiotic, Lactobacillus reuteri is one of the first "condition-specific" probiotics, designed and developed specifically to fight risk factors that lead to heart attacks, strokes, and other cardiovascular catastrophes [4].

The human microbiome project has opened the era of designing organ specific probiotics, which can be administered to susceptible individuals and thereby prevent the pathology to develop in that organ system and forms an essential part of regenerative medicine. Many such novel probiotics will boost healthcare and reduce cost of disease treatment and disease prevention.

\section{References}

1. Collins FS, Lander ES, Rogers J, Waterston RH (2004) Finishing the euchromatic sequence of the human genome. International Human Genome Sequencing Consortium Nature 431: 931-945.

2. Kar SK (2015) Translational Biomedicine: The Future of Modern Internal Medicine. Transl Biomed 6:36.

3. Jones ML, Martoni CJ, Parent M, Prakash S (2012) Cholesterol-lowering efficacy of a icroencapsulated bile salt hydrolase-active Lactobacillus reuteri NCIMB yoghurt formulation in hypercholesterolaemic adults. $\mathrm{Br} \mathrm{J}$ Nutr 107:1505-1513.

4. Jones M, Martoni C, Prakash S (2012) Cholesterol lowering and inhibition of sterol absorption by Lactobacillus reuteri NCIMB: a randomized controlled trial. Eur J Clin Nutr 66:1234-1241. 